\title{
Profiling Sprints using On-Body Sensors
}

\author{
S. Taherian, M. Pias, R. Harle, \\ G. Coulouris, and S. Hay \\ Computer Laboratory \\ Cambridge University, CB3 OFD, UK \\ $\{$ st344,mp431,rkh23,gfc22,sjeh3\}@cl.cam.ac.uk \\ J. Cameron and J. Lasenby \\ Department of Engineering \\ Cambridge University \\ Cambridge CB2 1PZ, UK \\ $\{j i c 23, j l 221\} @$ cam.ac.uk \\ G. Kuntze, I. Bezodis, G. Irwin, \\ and D. Kerwin \\ Cardiff School of Sport, UWIC \\ Cyncoed Campus, Cardiff, CF23 6XD, UK \\ \{gkuntze,ibezodis,girwin,dkerwin\}@uwic.ac.uk
}

\begin{abstract}
This paper describes the design, implementation and deployment of a wireless sensor system for athletes. The system is designed to profile sprints based on input from on-body sensors that are wirelessly connected to a nearby infrastructure. We discuss the choice and use of inexpensive Force Sensitive Resistors (FSRs) to measure foot event timings and provide a detailed analysis of the profiling method used to represent high-level information to the coaches and athletes.

In this profiling method, we detect sprinting intervals from high-resolution sensor data, and compute the ground contact times for sprinting performances. We validate our results using force plates and show that the system achieves comparable accuracy in measuring the foot contact times (millisecond accuracy) without the limitations of one or few steps.
\end{abstract}

Keywords-on-body sensor system; sprinting; sports profiling

\section{INTRODUCTION}

Wireless sensing technologies are becoming popular in the healthcare domain because they allow long-term monitoring of patients in their natural environment. This is particularly useful for the elderly, where constant supervision may be appropriate. There is, however, a growing interest in applying similar ideas at the other end of the dynamic scale: the athlete. Sports offer a challenging environment for sensing where sensor sampling rate and accuracy often takes precedence over battery lifetime.

To better understand the application of wireless sensing in sport, we developed a system to augment elite sprinters in their training environment. We shall describe the design and implementation of the high sampling-rate wireless system that allows data to be obtained from sensors attached to a sprinter's feet. The system has been used to profile sprints and derive ground contact times for use by coaches and athletes.

This paper is structured as follows. We first review the use of technology in sprint training, before deriving requirements for an on-body sensor system. We detail our design of such a system, and discuss the profiling algorithms used to deliver useful information to coaches and athletes. We then evaluate the system usage and the profiling accuracy. Finally, we review related work, identify future research and conclude.

\section{Technology In SPRINTING}

Training programmes for sprinting are composed of many elements, the largest of which pertains to technical training. In such training, the athlete seeks to improve the biomechanics of their actions in order to produce optimal movements in competition. Such training involves continual practice of the motion ('training specificity') and is typically composed of repetitions of sprints during which the coach observes and provides feedback to the athlete.

To date, the primary use of technology in this domain has been to assist performance analysis. High speed video cameras, light gates (which measure the crossing time of the athlete) and (rarely) optical motion capture systems have all been used. All of these systems are predominantly track-side, deployed as part of the infrastructure, with limited portability and coverage.

\section{REQUIREMENTS}

The requirements for any system deployed to augment sprint training are stringent: track performances are judged to within hundredths of a second and the movements are at the extremes of human capability. To gather requirements, we used a series of semi-structured interviews with a set of leading sprint coaches. The results are presented in [13] and identify four primary interests for sprinters: arm action, foot ground contact, hip height and running posture. Of these, details of the foot ground contact were highly prized because the information is not currently available. Coaches believed that ground contact time (the time a foot spends in contact with the track with each step) related strongly to the speed of the athlete.

A typical contact lasts approximately $100 \mathrm{~ms}$, and a $10 \%$ variation was believed to make a significant difference. Force plates embedded into a track have been used to measure these quantities, but a single force plate can only cover at most one contact due to its limited size and lack of portability (the track must be destructively modified to embed the plate). Coaches had not, therefore, seen a sequence of contact times corresponding to an entire repetition.

Optical motion capture systems have also been proposed to measure the contact times based on two markers on the foot, and these have been shown to have an accuracy of 


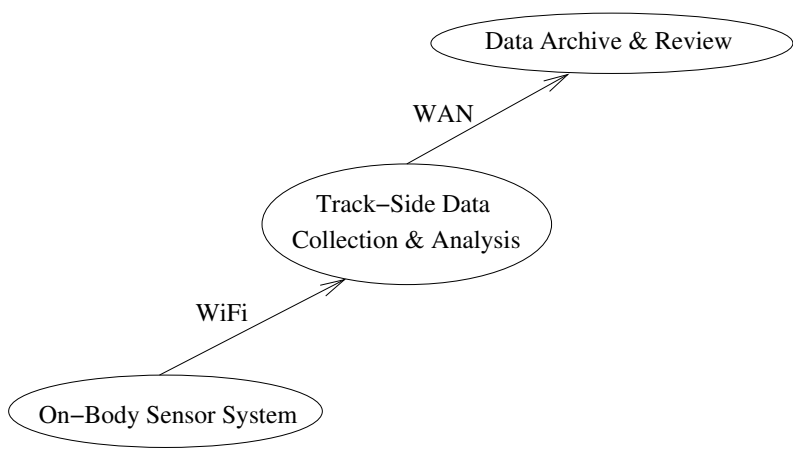

Figure 1. High-Level System Architecture

approximately $5 \mathrm{~ms}$ [5]. This error is too great given the expected variability. Additionally, motion capture systems are very expensive and typically only offer $20 \mathrm{~m}$ coverage.

In order to portably collect information on ground contact times over large areas, we sought to develop a system that used on-body sensors rather than rely on track-side infrastructure. We identified the following requirements:

- kHz-Sampling. The foot contact time is of the order of $100 \mathrm{~ms}$ and the variability across elite athletes is bounded by approximately $10 \mathrm{~ms}$, making sampling rates of $1 \mathrm{kHz}$ or greater necessary.

- Careful Ergonomics. With small biomechanical changes giving rise to large performance differences, any sensor system attached to the body must be lightweight and ergonomic. We have found that a $30 \mathrm{~g}$ mass is approximately at the limit of acceptability. Additionally, it needs to be firmly attached without adversely affecting performance.

- Robust Hardware. With limbs moving as fast as possible, any foot sensors will experience high accelerations and strong impulses. We have observed accelerations well in excess of $50 \mathrm{~g}$.

- Reliable Capture. Data capture can be considered one-shot since fatigue quickly sets in on a training sprinter. Real time status and control at the track-side is necessary.

- Long-term Data Review. Long-term cross-session analysis must be supported away from the training venue.

- Low Cost. The lower the system cost, the more likely it is to be deployed and used.

\section{SySTEM Design}

\section{A. Overall Architecture}

With robustness and reliability in mind, we simplified the system by supporting only a single sensor node at any given time. This removed the need for complex (and often fragile) time synchronisation of multiple nodes and reduced the weight and physical profile of the hardware. The system

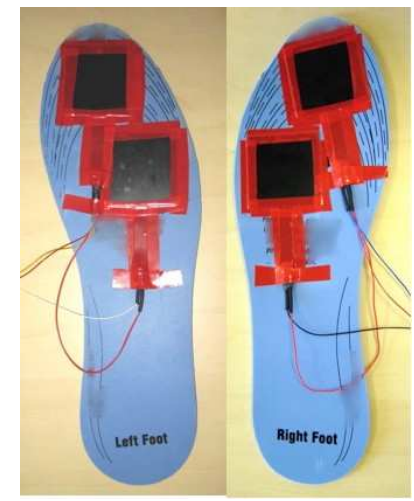

Figure 2. FSR-augmented insoles. The FSR sensors (black squares) are attached to the underside of the insoles.

itself was composed of three elements (Figure 1): an onbody sensor system; an in-field data collection and analysis tool and a software to archive and review data.

\section{B. On-Body Sensor System}

Sensors. Our first approach used accelerometers to isolate the moments of impact for touch-down and toe-off times. Although good for identifying the touch-down time (a sharp impact), accurately determining toe-off times proved near impossible because the foot is 'peeled' from the floor[6]. We also evaluated piezo-electric based sensors (PVDF films), ${ }^{1}$ but these exhibited poor durability and quickly became unreliable in continuous use.

Our final sensor choice was a set of Force Sensing Resistors (FSRs), ${ }^{2}$ see Figure 2. These use a polymer film which exhibits an extreme decrease in resistance under load. FSRs are cheap analogue sensors with very fast response times. Their simple design makes them highly durable and yet very flexible and lightweight.

Embedded Device. The on-body sensor node was based on a Crossbow Imote $2^{3}$ running Linux. This provided a relatively powerful CPU (Intel PXA271) capable of handling the high-rate sampling, whilst allowing rapid development on a familiar platform.

Attaching an Imote2 to an athlete such that it had no impact on the performance proved very difficult. Following experimentation, the lower calf and the lower back were identified as acceptable sites. When on the lower calf, the sensor-node wiring was simpler to route because it was shorter. However, this site limited us to sensing the contact times for a single foot. When monitoring both feet simultaneously, we used an elasticated pouch at the back of the running tights with wiring channels sewn down the legs.

Communications. In order to connect the track-side equipment to the on-body node, we chose WiFi. In addition

\footnotetext{
${ }^{1}$ http://www.msiusa.com/download/pdf/english/piezo/techman.pdf

${ }^{2}$ http://www.interlinkelectronics.com/

${ }^{3}$ http://www.xbow.com/Products/productdetails.aspx?sid=253
} 


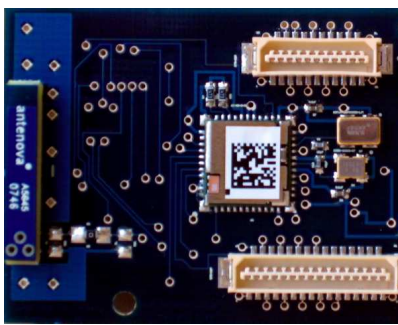

Figure 3. Custom Imote2 sensor board

to the benefits of inter-operability and implementation maturity, WiFi offers higher data rates and greater operational range compared to other protocols, alongside a power draw that is sustainable for the few hours of a training session.

We created a custom WiFi module for the Imote2 (which does not itself feature WiFi). The resultant daughterboard (Figure 3) used the Marvell $8686 \mathrm{WiFi}$ chip (with power management) and additionally featured a MAX1238 ADC to connect up to 12 FSR sensors. The design documents for the WiFi daughter board are available as open source. ${ }^{4}$

\section{Track-side Data Collection \& Analysis}

The track-side system fulfills three requirements: provides a low-rate live sensor feed to show that the system is working, reliably transfers the data and clears the on-body node's persistent storage, and stores data (along with the derived values) at an Archive Server. At present, we use a laptop to perform these operations. The laptop is wirelessly connected either to the Imote2 over a private WiFi network or to the building-wide WiFi when communication with the Archive Server is needed.

The laptop provides an interface to control the highresolution data recording at the on-body node. During recording, every sample is stored in a ring buffer in the Imote 2 memory and is periodically written to its flash disk when possible (the write speed attainable with such memory on the Imote2 is too slow to write directly to it). This minimises data losses should the Imote2 lose power for any reason. Once recording has ended, the data can be downloaded to the interface and saved to a file.

A profiling tool, described in the next section, analysis the entire dataset following data download to allow immediate performance review. These results, along with the raw data and suitable meta data are transferred to the Archive Server to enable offline data visualization and review.

\section{Data Archive \& Review}

In the data review phase, athletes and coaches alike displayed a strong interest in being able to review the data at their leisure. To allow this, and to increase accessibility in

\footnotetext{
${ }^{4}$ http://sourceforge.net/apps/mediawiki/imote2 linux/index.php?title=UCAM-WSB 100
}

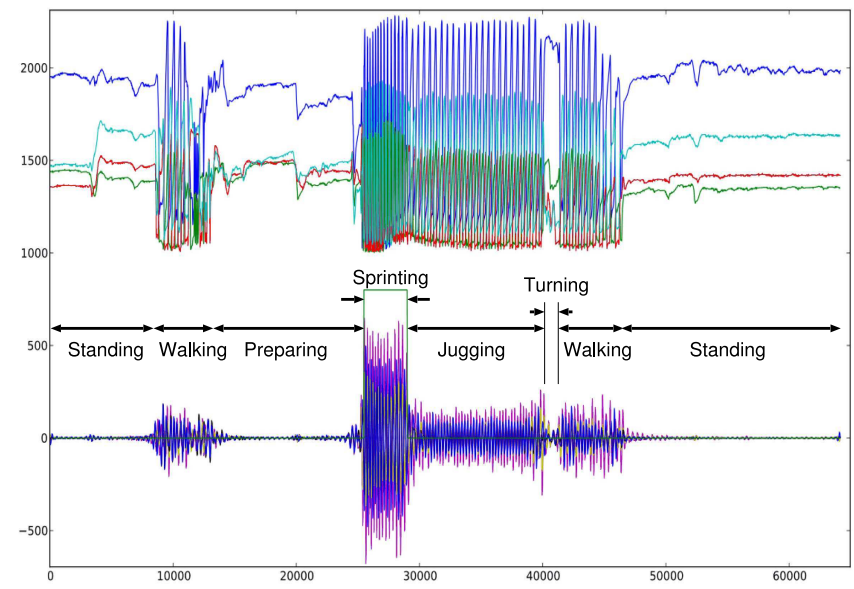

Figure 5. Sprint Interval Detection

general, we developed a web-based interface to allow data review (see Figure 4).

Profiling results direct the user to the sprinting sections of the data. A low-resolution summary graph (at the top) allows the user to select specific portions of the run to review in detail. Raw values are augmented with derived values such as contact times and flight times to assist the review and coaching.

\section{Profiling SPRINTS}

Recorded sessions capture numerous activities on the track, such as athlete walking, standing, preparing, and sprinting. Identifying the different classes of activities, from raw data, requires a trained eye (see Figure 5). In order to assist users, we developed two sets of algorithms that automatically segment and profile raw data and augment them with derived values as shown in Figure 4.

\section{A. Sprint Interval Detection}

The biomechanics of the sprinting motion is different than that of human walking, jogging, or jumping. It is important to identify and segment these motions to later profile them accurately. This process also reduces the profiling overhead and latency in generating derived values.

Sprinting is detected by identifying an interval of fast stepping sequence in the raw data. Figure 5 shows a recorded raw dataset (four FSR ADC counts) at the top, and a processed dataset (filtered FSR data) at the bottom. A Fast Fourier Transform is used to filter low frequencies $(<\approx 1.8 \mathrm{~Hz})$ and isolate the high frequencies $(\approx 1.8 \mathrm{~Hz}-2.6 \mathrm{~Hz})$ which relate to sprinting for each sensor stream. These signals are then combined, using SUM aggregation, to collectively indicate a region in which the highest stepping rate has occurred.

The bottom dataset in Figure 5 shows this reconstructed dataset, over which a combined MAX tumbling-window and threshold functions produce the final step-function that denotes the sprinting interval. The window size and threshold 




Figure 4. Visualiser

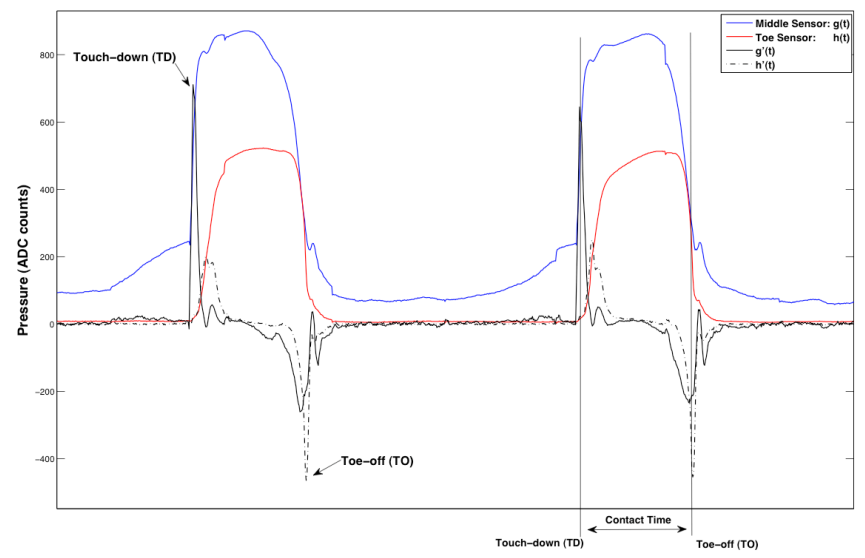

Figure 6. Example FSR sensor traces for a sprinter

cut-off value of $5 \mathrm{~ms}$ and $70 \%$ MAX, respectively, were determined empirically following the examination of 10 datasets. Only the output interval is further processed.

\section{B. Ground Contact Time}

Figure 6 shows the FSR traces for two sensors on a single insole for two sample foot contacts. Two timing events, touch-down (TD) and toe-off (TO), are important in extracting ground contact times for a run.

Figure 6 illustrates that each FSR signal exhibits a strong change on at least one of the events. To extract the event timings autonomously, we compute the first derivatives of the FSR signals (also shown on Figure 6). These signals highlight the signal changes that occur during the TD and TO events. The raw dataset is initially smoothed, using median and moving average filters of window sizes $75 \mathrm{~ms}$ and $5 \mathrm{~ms}$, to eliminate spikes and erroneous values prior to first derivative computations.

In the second phase of the algorithm, the derivative associated with the middle sensor is used to produce a set of candidate TD events, and the derivative associated with the toe sensor is used to produce a set of candidate TO events. Local maxima are used to detect TD events, and local minima are used to detect TO events. Each foot is processed separately to give two sets of candidate TD and TO events. In the third phase of the algorithm, we filter the false-positive events using a finite state machine which models the sprinting behavior.

Figure 7 shows a finite state machine which corresponds to the sprinting performance. The candidate events are filtered through this state machine to produce a final set of TD and TO events for each foot. An empirically determined minimum contact time duration of $80 \mathrm{~ms}$ is enforced for each foot. The contact times are then computed as the difference of the microsecond timestamps of each TD and its corresponding TO event. Trivially, the difference between 


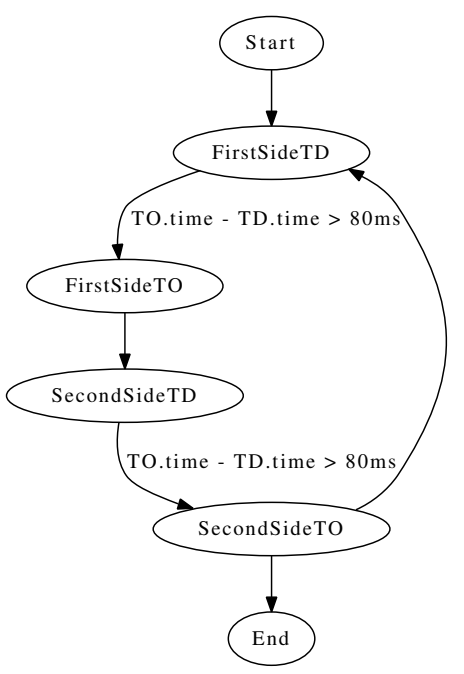

Figure 7. FSM for sprinting behavior

the step time and the contact time provides the flight time duration. These times are automatically extracted and plotted as shown in Figure 4.

\section{EVALUATion}

\section{A. General Feedback}

Athletes and coaches alike have expressed great interest in the system, and those that have trialled it have offered predominantly positive feedback. Coaches have been very keen to reduce the labour intensity of any of their tasks.

\section{B. Sprint Interval Detection}

The sprint interval detection algorithm has been applied to over 20 runs from 4 different athletes. Identified sprints have been visually confirmed (from the raw datasets) using a trained eye; and in several instances; they have also been confirmed using externally collected data sources (hand-held video camera data streams). These intervals have been successfully profiled using the described contact time algorithm.

\section{Contact Time Accuracy}

We evaluated the system using force plates, which are the standard method by which to measure contact times in the sports science community. Whilst a force plate is considered as a highly accurate measurement method for contact times, it can only capture a single foot contact per repetition, making the collection of a large volume of comparison data infeasible. This limitation was, of course, one of the primary motivations for our on-body sensor system.

In total we captured 38 foot contacts using both our wireless system $(2 \mathrm{kHz})$ and a force plate $(1 \mathrm{kHz}): 12$ were measured at the first contact out from the starting blocks (expected to be relatively lengthy contacts); 12 were collected from the fifth contact (expected to be shorter contact times); and 14 were collected from later in the

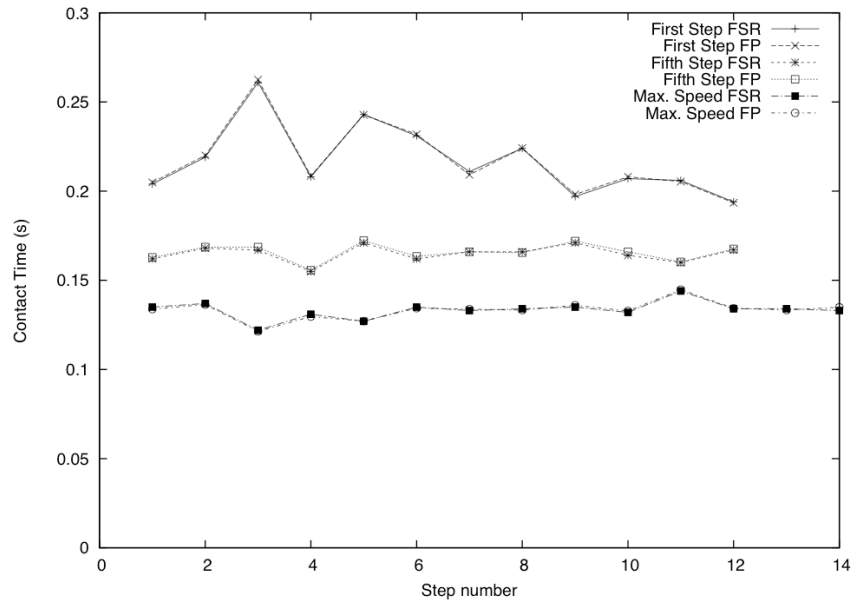

Figure 8. Computed contact times using FSR sensors and using a force plate (FP).

run, with the athlete at near maximal speed (expected to be shorter still).

Figure 8 shows the contact times computed using the force plate data and the FSR data. We observe that the differences between the force plate computation and the FSR computation are generally sub-millisecond. Due to the discussed limitations of the force plate, there were insufficient data to perform a detailed statistical error analysis, but we provide the following indicative metrics:

\begin{tabular}{|l|c|}
\hline RMS Error & $0.977 \mathrm{~ms}$ \\
Minimum Error & $0.01 \mathrm{~ms}$ \\
Maximum Error & $1.98 \mathrm{~ms}$ \\
\hline
\end{tabular}

VII. RELATED WORK

The sports domain has received relatively little attention in ubiquitous computing when compared to the healthcare and military domains (where BSNs are commonly used). This is despite publications highlighting the domain for pervasive computing [7].

Many sports-related pervasive publications shy away from the analysis of sporting technique since this requires high sensor resolution and sampling rates. A notable exception is the work by Michahelles et al., who applied Body Sensor Network techniques to skiing [11]. They used FSR sensors beneath the skier's feet in a similar configuration to the work described in this paper. However, they concentrated on using the magnitude of the response rather than identifying key events. The entire system was designed to capture data without a wireless link to fixed infrastructure and the resultant logging unit was considerable both in size and weight. This was acceptable to skiers (who rely less on range of movement) but would be intolerable for most other sports.

Paradiso at al. described the use of a wireless system for dancers [1], [2] that is used to measure the synchronization of complex movements. They used a small sensor node 
strapped to the wrist that transmitted accelerometer data using a custom radio channel. Wireless range was not cited as an issue, presumably because the dancing took place within a relatively small space. Subsequent work improved the system design and provided preliminary results when applied to baseball players [3].

With respect to systems used to measure contact times, Purcell et al. trialled the use of accelerometers sampled at $250 \mathrm{~Hz}$ and attached to the shin [12]. They used a Bluetooth connection to the athlete and reported ranges of up to $200 \mathrm{~m}$ and contact time accuracies of the order of a few milliseconds. However, the authors openly admit the results were preliminary and we have been unable to reproduce them.

In addition, researchers in the biomechanical and rehabilitation fields have made use of modified insoles to sense pressure [9], [8], [10], [4]. Whilst relevant, these systems have tended to concentrate on accurate force measurements using expensive sensors and capture equipment that, whilst portable, is too bulky for sports people (intended instead for gait analysis).

\section{CONCLUSIONS AND FURTHER WORK}

This paper has documented our experiences in creating a wireless sensor system for the sports domain. We have discussed the requirements, system architecture, algorithms, and evaluated the final result. In doing so we have made the following contributions.

- We have identified a series of requirements that are specific to the sprinting exercise.

- We have created a complete system able to profile sprints with a comparable accuracy but far greater range than the presently accepted equipment.

- We have created a web-based system to give ubiquitous access to the collected and analyzed data.

In general, our experiences working with coaches and athletes have highlighted that the sporting domain is a fertile application domain for sensor systems. We intend to continue working in the domain, and have identified a series of goals for the next version of our system:

- Synchronisation with Track-Side Sensors. Coaches have expressed interest in overlaying data sensed onbody with off-body data from e.g. video cameras.

- True Environment Integration. We feel strongly that the system should be deployed long-term in a training environment and operated solely by coaches or athletes (i.e. without expert intervention). Feedback will then permit refinement of the control and review interfaces.

\section{REFERENCES}

[1] R. Aylward and J. Paradiso. Sensemble: A wireless, compact, multi-user sensor system for interactive dance. In Proc. of NIME 2006, The 2006 International Conference on New Interfaces for Musical Expression, pages pp. 134-139, Paris, France, June 2006.
[2] R. Aylward and J. Paradiso. Poster paper: A compact, high-speed, wearable sensor network for biomotion capture and interactive media. In Proc. of the Sixth International IEEE/ACM Conference on Information Processing in Sensor Networks (IPSN 07), pages pp. 380-389, Cambridge, MA, april 2007.

[3] R. Aylward and J. A. Paradiso. A compact, high-speed, wearable sensor network for biomotion capture and interactive media. In IPSN '07: Proceedings of the 6th international conference on Information processing in sensor networks, pages 380-389, New York, NY, USA, 2007. ACM.

[4] S. J. M. Bamberg, A. Y. Benbasat, D. M. Scarborough, D. E. Krebs, and J. A. Paradiso. Gait analysis using a shoe-integrated wireless sensor system. IEEE Transactions on Information Technology in Biomedicine, 12(4):413-423, 2008.

[5] I. Bezodis, A. Thomson, M. Gittoes, and D. Kerwin. Identification of instants of touchdown and take-off in sprint running using an automatic motion analysis system. In H.-J. Menzel and M. Chargas, editors, Proceedings of the XXVth Symposium of the International Society of Biomechanics in Sports, pages pp 501-504, Ouro Preto, Brazil, 2007.

[6] L. Cheng and S. Hailes. On-body wireless inertial sensing for foot control applications. In Proceedings of IEEE International Symposium on Personal, Indoor and Mobile Radio Communications (PIMRC), Cannes, France, Sept. 2008.

[7] E. Chi, G. Borriello, G. Hunt, and N. Davies. Guest editors' introduction: Pervasive computing in sports technologies. Pervasive Computing, IEEE, 4(3):22-25, July-Sept. 2005.

[8] F. Tokita, K. Yamakoshi, T. Sasaki, and S. Ishii. Portable instrument for accurate measurement of plantar force distribution during dynamic activities. Medical and Biological Engineering and Computing, 33(4), 1995.

[9] A. Forner-Cordero, H. J. F. M. Koopman, and F. C. T. van der Helm. Inverse dynamics calculations during gait with restricted ground reaction force information from pressure insoles. Gait \& Posture, 23(2):189-199, February 2006.

[10] JJ Wertsch, JG Webster, and WJ Tompkins. A portable insole plantar pressure measurement system. Journal of Rehabilitation Research and Development, 1992.

[11] F. Michahelles and B. Schiele. Sensing and monitoring professional skiers. IEEE Pervasive Computing, 4(3):40-46, 2005.

[12] B. Purcell, J. P. Channells, D. A. James, and R. Barrett. Use of accelerometers for detecting foot-ground contact time during running. In Proceedings of SPIE on CD-ROM: Microelectronics, MEMS, and Nanotechnology, 2006.

[13] A. Thomson, I. Bezodis, and J. R.L. An in-depth assessment of expert sprint coaches' technical knowledge. Journal of Sports Sciences, 2009. 